This item is the archived peer-reviewed author-version of:

\title{
The benefit of design of support architectures for zeolite coated structured catalysts for methanol-to-olefin conversion
}

\section{Reference:}

Lefevere Jasper, Gysen Marijn, Mullens Steven, Meynen Vera, van Noyen J..- The benefit of design of support architectures for zeolite coated structured catalysts for methanol-to-olefin conversion

Catalysis today - ISSN 0920-5861 - 216(2013), p. 18-23

Full text (Publishers DOI): http://dx.doi.org/doi:10.1016/j.cattod.2013.05.020

To cite this reference: http://hdl.handle.net/10067/1105620151162165141 


\section{The benefit of design of support architectures for zeolite coated structured catalysts for methanol-to-olefin conversion}

4 Jasper Lefevere ${ }^{\mathrm{a}, \mathrm{b}}$, Marijn Gysen ${ }^{\mathrm{a}}$, Steven Mullens ${ }^{\mathrm{a}}$, Vera Meynen ${ }^{\mathrm{b}}$, Jasper Van Noyen ${ }^{\mathrm{a},{ }^{*}}$

a Sustainable Materials Management, Flemish Institute for Technological Research - VITO,

7 Boeretang 200, B-2400 Mol, Belgium

$8{ }^{b}$ Department of Chemistry, University of Antwerp,Universiteitsplein 1, B-2610 Antwerp, Belgium

9 *To whom correspondence should be addressed. Tel.: 0032-14-335672; Fax: 0032-14-321186;

10 E-mail: jasper.vannoyen@vito.be

\section{Abstract}

14 Zeolites are commonly used as catalysts for a wide range of industrial applications.

15 Macroporous supports with a thin active layer of zeolite make it possible to avoid a 16 trade-off between pressure drop and mass- and heat transfer. The architecture of these 17 porous supports defines the mass- and heat transfer properties of the catalyst. By 18 innovative three dimensional fiber deposition different architectures for the catalyst 19 support can be manufactured rapidly. In this work a wash coating method was 20 optimized for the coating of ZSM-5 on different types of support. The manufactured 21 catalysts were evaluated for the conversion of methanol to light olefins. The effect of 22 architecture of the support structure on the selectivity and activity of the reaction is 23 demonstrated.

25 Keywords: MTO, ZSM-5, structured catalyst, 3DFD

29 In terms of production volume, ethylene and propylene are among the most important

30 intermediates in the chemical industry. As the worldwide demand for these light olefins

31 increases year after year, there is an on-going search for alternative production 
32 technologies for ethylene and propylene. Nowadays, thermal cracking of naphtha is the

33 main production process for these light olefins. Due to the increase in crude oil prices

34 and a fast growth of the world propylene demand, new processes for olefin production

35 using alternative feedstocks receive increasing attention. A high selectivity towards

36 propylene is favourable in these new processes, as the market for propylene grows even

37 faster than that for ethylene [1,2]. One alternative for the steam cracking process is the

38 production of light olefins out of methanol (methanol-to-olefins, MTO). Methanol can

39 be produced with proven and mature technologies such as steam reforming of natural

40 gas or gasification of coal using syngas technology [3]. The MTO technology is a very

41 promising way of converting alternative feedstock such as natural gas and coal to

42 chemicals and fuels with methanol as an intermediate. The MTO technology has been

43 proven successful with a first commercial plant start-up in China in 2010, and more

44 being constructed $[4,5]$.

45 Zeolites are, to the best of our knowledge, the only type of catalysts that are currently

46 being used in industrial MTO processes. Two types of zeolite are effectively used: SAPO-

4734 by UOP/Ineos and Dalian Institute of Chemical Physics, and ZSM-5 used in the Lurgi

48 process $[5,6]$. SAPO-34 is a silicoaluminophosphate with small 8 membered pores, and

49 has been proven to have a good selectivity to light olefins. However, it shows fast

50 deactivation due to coke formation [7]. On the other hand, the ZSM-5 catalyst, a

51 medium-pore zeolite composed of intersecting straight (5.3 $\times 5.6 \AA$ ) and sinusoidal (5.1

$52 \times 5.5 \AA ̊$ Å) 10-ring pores, generally has a much better stability [8]. ZSM-5 however shows

53 inferior selectivity for ethylene and propylene as compared to SAPO-34 [4]. Due to its

54 larger pores the selectivity towards heavier products such as butylene is higher for the

55 ZSM-5 catalyst. This catalyst is generally shaped as microgranules or extruded pellets

56 and used in a random packed bed. These packed beds have a number of disadvantages,

57 including limited heat- and mass transfer, high pressure drop and unevenly distributed

58 flows, leading to differences in contact time and loss of selectivity. In recent years, the

59 interest for zeolite coated structured catalysts has been growing as they allow a low

60 pressure drop in combination with high mass- and heat transfer and controlled 
61 residence times. The zeolite coating can be prepared using different techniques such as

62 wash coating [9], and hydrothermal coating [10-17] or a combination of both [18]. A

63 broad spectrum of porous or non-porous support materials can be used, such as $\mathrm{Al}_{2} \mathrm{O}_{3}$

64 [11], glass [12], stainless steel [10,13], alumina ceramic foam [14], cordierite honeycomb

$65[15,16,19]$, or SiC $[17,20,21]$.

66 The architecture of the support is of crucial importance for the mass- and heat transfer,

67 pressure drop and contact time. By "robocasting" or 3 dimensional fiber deposition

68 (3DFD) an optimal architecture can be manufactured, combining good mass transfer

69 with low pressure drop [22-25]. This technique comprises a layer by layer build-up of a

70 ceramic or metallic support structure. With the 3DFD technique an unprecedented

71 freedom of design and degree of control over the architecture is possible, allowing

72 optimization for different applications [26]. Rapid prototyping of ceramic and metallic

73 supports allows an almost unlimited amount of unique architectures with an excellent

74 reproducibility. By combination of modelling and testing, a fast and good choice of

75 supports with adequate mechanical properties and low pressure drop can be achieved 76 [25].

77 In this study, the influence of different architectures of the 3DFD support structures on

78 the conversion of methanol to light olefins has been studied. A comparison has been

79 made with cordierite honeycombs and a packed bed of pelletized ZSM-5 powder. The

80 influence of the support architecture on the catalytic properties of the zeolite has been

81 studied. First, the wash coating process of ZSM-5 on the structured stainless steel

82 supports was optimized and characterised with regard to coating adhesion. Then, the

83 coated structured catalysts have been evaluated for their catalytic performance in the

84 methanol dehydration and MTO reaction. The effect of architecture of the support

85 structure on the selectivity and activity of the reaction is demonstrated.

\section{Experimental}

\subsection{Preparation of the catalysts}


90 The stainless steel (316L) support structures were prepared using 3DFD technology, as

91 described in literature [27,28]. 3DFD structures with 1-1 and 1-3 stacking were

92 manufactured using a $0.9 \mathrm{~mm}$ nozzle and a programmed inter fibre distance of $1.1 \mathrm{~mm}$.

93 The 1-1 stacked 3DFD structure has straight channels in the direction of the flow (1.1 $\mathrm{x}$

$941.1 \mathrm{~mm})$ in combination with smaller radial channels $(1.1 \times 0.45 \mathrm{~mm})$ in the two

95 directions (Table 1). The 1-3 stacked 3DFD structure consists out of zigzag channels in

96 the direction of the flow $(1.1 \times 1.1 \mathrm{~mm})$ and smaller straight channels $(1.1 \times 0.45 \mathrm{~mm})$ in

97 the two radial directions. The fibers of the 1-1 structure show a tetragonal symmetry

98 while the 1-3 stacked structure shows a face centered symmetry (See supplementary

99 information) [29]. After synthesis, the stainless steel 3DFD samples were sintered at

$1001300^{\circ} \mathrm{C}$ for 4 hours. The monoliths used in the comparison were commercially available

101 cordierite honeycomb structures with 400 cells per square inch (cpsi) and a wall

102 thickness of $6.5 \mathrm{mil}(0.165 \mathrm{~mm})$ (NGK Insulators). The honeycomb structures have

103 straight channels with a size of $1 \times 1 \mathrm{~mm}$ and no radial channels. The support structures

104 were cut into cylinders with a diameter of $21 \mathrm{~mm}$ and a length of $25 \mathrm{~mm}$. All supports

105 were cleaned in acetone, ethanol and distilled water for 10 minutes under ultrasonic

106 conditions and dried overnight at $100^{\circ} \mathrm{C}$.

107 The coating slurry was prepared as follows: ZSM-5 (TZP302, Süd-chemie, Si/Al ratio 25,

$108 \mathrm{~d}_{50} 8.77 \mu \mathrm{m}$ ) powder was dispersed in distilled water and colloidal silica (LUDOX HS-40,

109 Sigma-Aldrich) was added. The final composition of the wash coating slurry consisted of

$11030 w t \%$ ZSM-5, 1wt\% methylcellulose and 1wt\% LUDOX HS-40.

111 The support structures were coated by a wash coating procedure. The structures were

112 placed vertically and the coating slurry was perfused through the substrate using a

113 peristaltic pump. After a contact time of 10 seconds at which the slurry remained

114 immobile, the excess of slurry was discharged using an air flow. The coated structures

115 were dried overnight at $100^{\circ} \mathrm{C}$. Finally, the samples were calcined for 3 hours at $550^{\circ} \mathrm{C}$

116 with a heating rate of $60^{\circ} \mathrm{C} / \mathrm{h}$ in ambient atmosphere to remove all organic compounds

117 from the coating.

118 
120 Rheology was used to determine the viscosity of the slurry as a function of the shear

121 rate (kinexus rheometer, Malvern Instruments, Worcestershire, United Kingdom). Shear

122 rates were varied between 0.01 and $1000 \mathrm{~s}^{-1}$ at a temperature of $25^{\circ} \mathrm{C}$.

123 Particle size distribution was measured using laser diffraction (Mastersizer X, Malvern

124 Instruments, Worcestershire, United Kingdom) using a beam length of $10 \mathrm{~mm}$.

125 The amount of zeolite deposited was calculated from the weight increase after 126 calcination. The adhesion strength of the coating onto the stainless steel supports was

127 evaluated by resistance to ultrasonic treatment. The dried samples were weighed

128 before and after treatment in an ultrasonic bath during 1 minute. The weight loss of the

129 coating gives an indication of the adhesion strength of the coating to the structured

130 support $[30,31]$.

131 The apparent specific surface area of the different catalysts was measured by $\mathrm{N}_{2}$

132 sorption at $-196^{\circ} \mathrm{C}$ using the BET method (Autosorb-1, Quantachrome, Germany). Prior

133 to $\mathrm{N}_{2}$ sorption measurements, the samples were outgassed for $16 \mathrm{~h}$ at $200^{\circ} \mathrm{C}$ in order to

134 remove all adsorbed water from the zeolite.

135 X-ray diffraction (XRD; X'pert PRO, Philips, Eindhoven, The Netherlands) was used to

136 examine the phase and crystallinity of the coating using a Cu-K $\mathrm{X}$-ray source $(\lambda=$ $1371.54056 \AA$ A).

138 The surface and cross-sections of the catalyst were observed using a cold field emission 139 scanning electron microscope (FEG-SEM) type JSM6340F (JEOL, Tokyo, Japan) at an 140 acceleration voltage of $5 \mathrm{keV}$. To avoid charging under the electron beam during SEM, 141 all samples were coated with a thin $\mathrm{Pt}(80) / \mathrm{Pd}(20)$ (surfaces) or Au (cross-sections) layer 142 ( 1.5 nm), using a Cressington 208 HR (UK) and a Balzers Union SCD 040 (Balzers, 143 Liechtenstein) high resolution sputter-coater, respectively. The thickness of the coating 144 deposited onto the fiber was observed.

$145 \mathrm{NH}_{3}$-Temperature Programmed Desorption ( $\mathrm{NH}_{3}$-TPD) was performed on the pure 146 zeolite powder and the coating to measure their acidity (Autosorb-iQ-Chemi, 147 Quantachrome, Germany). Prior to TPD measurements, the samples were outgassed for 
$14816 \mathrm{~h}$ at $200^{\circ} \mathrm{C}$ under vacuum in order to remove all adsorbed water from the zeolite.

149 After the pre-treatment the samples were saturated with ammonia at $100^{\circ} \mathrm{C}$. The excess

150 of ammonia was removed with a helium flow for 30 minutes. The temperature was

151 raised from $100^{\circ} \mathrm{C}$ to $750^{\circ} \mathrm{C}$ at a rate of $10^{\circ} \mathrm{C} / \mathrm{min}$ for the desorption of ammonia. The

152 desorbed ammonia was detected using a thermal conductivity detector (TCD).

\subsection{Catalytic testing and reaction data analysis}

155 Catalytic testing was performed in a fixed bed reactor with an inner diameter of $25 \mathrm{~mm}$

156 and a length of $300 \mathrm{~mm}$ at atmospheric pressure and temperatures of $350^{\circ} \mathrm{C}$. The 157 catalyst structures with a length of $25 \mathrm{~mm}$ were packed in thin layer of quartz wool and 158 placed in the middle of the reactor. The zeolite powder used for the comparison was 159 pelletized and sieved (0.125-0.250 $\mathrm{mm}$ fraction). The pelletized particles were diluted 160 with inert, sintered $\mathrm{Al}_{2} \mathrm{O}_{3}$ pellets and placed in the same reactor volume as the 161 structured catalysts. Table 1 gives an overview of the different catalysts tested.

165 Nitrogen gas was co-fed $(300 \mathrm{ml} / \mathrm{min}$ ) and used as diluent for methanol (Merck, $\geq 99.9$ $166 \%$ ). The reaction was carried out at a weight hourly space velocity (WHSV) in the range 167 of $4.56-36.46 \mathrm{~h}^{-1}$. The product distribution has been analyzed with a gas chromatograph 168 using a dual thermal conductivity detector and flame ionization detector (450-GC,

169 Bruker, Bremen, Germany). After achieving equilibrium at each flow rate an isothermal 170 period of 1 hour was kept and 4 gas measurements were performed. After each run the 171 catalyst samples were regenerated by burning of the coke at $550^{\circ} \mathrm{C}$ for 2 hours in a 172 stream of air. In the data analysis all $\mathrm{C}_{5}+$ species are combined in one group as this is not 173 the main focus of this research.

174 The conversion of methanol was calculated as:

$$
X_{\mathrm{MeOH}}=1-\frac{C_{\mathrm{MeOH}, \text { outlet }}}{C_{\mathrm{MeOH}, \text { inlet }}}
$$


176 The selectivity and yield of the different components was defined as:

177

202 Further improvement of the coating adhesion was made by milling the zeolite before 203 the coating process [34]. The initial zeolite powder had a $d_{50}$ (50\% of the particles

with $\mathrm{n}$ representing all components in the product stream except methanol and DME.

\section{Results and discussion}

\subsection{Preparation and characterization of the catalysts}

Initially, a coating slurry was used containing 30 wt\% ZSM-5, 1 wt\% methylcellulose and $69 \%$ distilled water. After wash coating and calcination, the adhesion of this coating was evaluated. After 1 minute of ultrasonic treatment, $47 \%$ of the coating was removed from the structure. A weight loss of maximum 5 wt\% of the coating was targeted as this is required for catalytic applications [32]. Therefore, colloidal silica was added as a binder to the coating suspension which improved the adhesion to the support (Figure 1). A decrease of the weight loss was observed from $47 \%$ loss without silica addition down to $5 \%$ loss at 2 wt\% silica. In addition to the better adhesion, the amount of zeolite deposited increases with higher colloidal silica concentrations, from $0.1 \mathrm{~g} / \mathrm{cm}^{3}$ with no silica to $0.18 \mathrm{~g} / \mathrm{cm}^{3}$ with 2 wt\% silica. Rheology measurements show a shear-thinning effect for all coating suspensions with an increase of the viscosity for higher silica concentrations especially at low shear rates (Figure 2). This effect can explain the higher loadings deposited with increasing silica concentration [33]. It seems that the adhesion of the zeolite particles to each other and to the support is improved by the colloidal binder due to an increased contact surface between the large particles themselves and the particles and support. With increasing colloidal silica concentration the amount of particles in the suspension rises so more silica particles hold the zeolite particles together, leading to a coating with better adhesion. At $2 \mathrm{wt} \%$ silica the viscosity is so high that removal of the excess wash coat from the porous substrate and thus the uniformity of the coating becomes an issue, so in further testing 1 wt\% silica is used.

$$
S_{i}=\frac{C_{i}}{\Sigma_{n} C_{n}} \quad y_{i}=S_{i} * X_{M e O H+D M E}
$$


204 smaller then this size) of $8.77 \mu \mathrm{m}$ and $d_{90}$ (90\% of the particles smaller then this size) of

$20514.1 \mu \mathrm{m}$. By planetary ball milling (250 rpm using $3 \mathrm{~mm}$ zirconia beads), the $\mathrm{d}_{50}$ reduced

206 to $3.40 \mu \mathrm{m}$ after 15 minutes of milling and $2.73 \mu \mathrm{m}$ after 30 minutes of milling. After 30

207 minutes the $d_{90}$ was reduced to $10.01 \mu \mathrm{m}$. The particle size distribution showed a

208 bimodal distribution with a first peak of particles smaller than $2 \mu \mathrm{m}$ and a second peak

209 with particles between 2 and $10 \mu \mathrm{m}$. XRD measurements show a slight decrease in

210 crystal size by milling from $63.0 \mathrm{~nm}$ before milling to $53.2 \mathrm{~nm}$ after milling. Rheology

211 measurements confirm that the larger amount of smaller particles in the coating slurry

212 after milling results in higher viscosity at low shear rate (data not shown). Figure 3

213 shows the beneficial effect of milling on the adhesion of the coating. The combination of

214 the effect of milling and an addition of $1 \mathrm{wt} \%$ of silica leads to a further improvement of

215 the coating adhesion (Figure 3). The amount of zeolite deposited before ultrasonic

216 treatment increases by milling if no silica is present. At a concentration of $1 \%$ of silica

217 the loading does not increase by milling. It is supposed that smaller zeolite particles

218 have a better anchoring at the rough surface of the support leading to a better

219 adhesion. After these modifications, the weight loss during ultrasonic treatment (1

220 minute) has been reduced to $4 \%$. At longer ultrasonic treatment (10 minutes) the

221 weight loss of the coating only slightly increased up to $6 \%$. These structures with stable

222 coating were used for catalytic testing (Figure 4). Furthermore, by repeated coating the

223 loading of the structures could be increased from 0.1 to $0.4 \mathrm{~g} / \mathrm{cm}^{3}$. XRD measurements

224 confirm that the crystallinity of the ZSM-5 is preserved in the coating (See

225 Supplementary Information). Moreover $\mathrm{N}_{2}$ sorption shows similar apparent surface

226 areas for the pure zeolite as for the coated zeolite (pure zeolite $434 \mathrm{~m}^{2} / \mathrm{g}$ compared to

$227428 \mathrm{~m}^{2} / \mathrm{g}$ of the coated layer). $\mathrm{NH}_{3}$-TPD measurements of the pure zeolite and the

228 coating confirm that all acid sites on the zeolite surface are preserved during the coating

229 process and that no pore blocking occurs (See Supplementary Information). The

230 distribution of strong and weak acid sites on the zeolite surface remains similar after the

231 wash coating process (See Supplementary information) and is in line with literature

232 values [31,35]. 


\section{$234 \quad 3.2 .1$ Methanol dehydration}

235 At low temperatures $\left(250^{\circ} \mathrm{C}\right)$ the dehydration of methanol to dimethylether (DME) is the

236 main reaction. As this reaction is exothermic, structured catalysts can be very use-full

237 for efficient removal of the heat of the reaction. This could be beneficial in applications

238 were high yield of dimethylether is desired such as DME as transportation fuel. In table

2392 the results of the catalytic conversion of methanol at $250^{\circ} \mathrm{C}$ with a WHSV of $4.56 \mathrm{~h}^{-1}$

240 are given. The reaction data show a high selectivity (over $99 \%$ ) towards DME at $250^{\circ} \mathrm{C}$.

241 All structured catalysts show similar behavior, reaching a conversion of $84.5 \%$, about

$24220 \%$ higher than for the packed bed. These results indicate a higher effectiveness of the

243 catalyst in the coating. These structured types of catalyst show promising results for the

244 methanol dehydration reaction. The stability of this high DME yield was not tested as

245 this was not within the scope of this paper but could be of interest for future work.

\section{$248 \quad$ 3.2.2 Methanol-to-olefins}

249 At higher temperatures the selectivity of the reaction shifts to heavier products such as 250 olefins, alkanes and aromatics. The conversion of methanol was monitored at $350^{\circ} \mathrm{C}$ and 251 different WHSVs between 4.56 and $36.48 \mathrm{~h}^{-1}$ (Figure 5). In the results of this catalytic 252 testing dimethylether is included in the reactant pool and not considered as a product. 253 The results show that the conversion of the structured catalysts is higher than that of 254 the packed bed at all tested WHSVs. At the lowest WHSV of $4.56 \mathrm{~h}^{-1}$ the packed bed 255 gives $84.60 \%$ conversion while the structured packing show full conversion of methanol. 256 It was already suggested by Patcas [36] that the reason for the higher effectiveness of 257 the coated structures over a packed bed is due to diffusional transport limitations. As 258 the bulk phase of the packed bed pellets is larger and has a higher density than the 259 coating, the effectiveness of the coating is higher because of internal diffusion limitation 260 in the pellets of the packed bed. At higher WHSV the conversion of the packed bed 261 progressively declines. The catalyst deposited onto the surface of a support is more 
262 effective at converting methanol and DME. All structured catalysts reach $100 \%$

263 conversion at WHSV from 4.56-9.12 $\mathrm{h}^{-1}$. The conversion of the ZSM-5 coated 3DFD 1-1

264 catalyst starts to drop at a WHSV of $18.24 \mathrm{~h}^{-1}$ while the conversion of the honeycomb

265 and 3DFD 1-3 sample only start to decline at a WHSV of $27.35 \mathrm{~h}^{-1}$. Interestingly, at very

266 high WHSV the conversion of the 3DFD 1-3 sample is higher than all other samples,

267 which can probably be ascribed to better mass- and heat transfer properties of this

268 structure $[22,23,25]$.

270 The combined ethylene and propylene yield was studied at different WHSVs at $350^{\circ} \mathrm{C}$

271 (Figure 6). The results show that the 3DFD 1-3 sample achieves the highest light olefin 272 yield of all tested samples at a WHSV of $27.35 \mathrm{~h}^{-1}$ and $350^{\circ} \mathrm{C}$. At the same conditions and 273 comparable conversion the 1-3 3DFD achieves higher ethylene and propylene yield than 274 the straight channel honeycomb. In the honeycomb catalyst the formation of heavier 275 products is higher. It is proposed that the better mass transfer of the 1-3 3DFD structure 276 leads faster evacuation of the light products and so better selectivity. The catalytic 277 testing also suggests that at different reaction conditions there is a different optimal 278 catalyst for this reaction. It is suggested that the different mass- and heat transfer 279 properties of the catalytic structures lead to different optimal operating conditions. 280 These results also suggest that the highest olefin yield is achieved at below $100 \%$ 281 conversion. Indeed figure 7 shows that the ethylene/propylene selectivity increases with 282 decreasing conversion. At an equal conversion (80\%) of all catalysts, the 3DFD type 283 samples achieve the highest ethylene and propylene selectivity. The honeycomb catalyst 284 shows a higher selectivity towards butylene, $\mathrm{C}_{2}-\mathrm{C}_{4}$ alkanes and larger $\mathrm{C}_{5}{ }^{+}$products at 285 WHSVs ranging from $4.56 \mathrm{~h}^{-1}$ to $27.35 \mathrm{~h}^{-1}$ than the 3DFD structured catalysts. The packed 286 bed shows a higher tendency for the undesired formation of $\mathrm{C}_{2}-\mathrm{C}_{4}$ alkanes at all WHSVs 287 tested compared the structured catalysts (See supplementary information). 


\section{Conclusions}

292 The use of rapid prototyping techniques such as three dimensional fiber deposition

293 allows fast manufacturing of highly reproducible supports with different architectures.

294 The optimized coating method enables the deposition of a homogenous catalytic layer

295 of zeolite on the surface of the support. By addition of colloidal silica and milling of the

296 zeolite, the deposited coatings show a good adhesion to the surface of the support, due

297 to a better interaction with the support surface and interaction between the zeolite

298 particles. A hierarchical porous catalyst is obtained after coating by the combination of

299 the macroporous support with the microporous zeolite layer.

300 The ZSM-5 coated structures were benchmarked for the conversion of methanol to 301 dimethylether and to olefins. The influence of the architecture on the catalytic 302 performance of the zeolite was studied rather than the intrinsic properties of the zeolite 303 catalyst. At low temperature the catalytic testing indicates that high selectivity towards 304 DME can be achieved using a structured catalyst with straight channels. The contact 305 time distribution is of key importance to achieve high DME selectivity. At $350^{\circ} \mathrm{C}$ the 306 structured catalyst are effective in converting methanol to olefins even at high methanol 307 feed rates. It was shown that a 3DFD support structure with 1-3 architecture, having 308 tortuous channels, exhibits the highest yield of light olefins at high WHSV. The main 309 reason for this is the better mass and heat transfer properties of this type of structured 310 catalyst.

311 Further improvement of the catalyst structures can be made by optimization of the fiber

312 thickness and spacing between the fibers of the 3DFD structure. This could lead to an 313 even more effective catalytic layer. In future work the stability of these catalyst in a 314 relation to the heat and mass transfer properties of different architecture structured 315 catalyst will be considered.

316 By using innovative rapid prototyping techniques, combined with an optimized wash 317 coating method, new structured catalysts have been manufactured. These novel 318 catalysts have been compared with packed bed and a honeycomb type support for the 319 conversion of methanol at different temperatures, and show excellent catalytic 
properties. This work demonstrates that the optimal catalyst structure depends on the application and the reaction conditions used. Rapid prototyping allows fast synthesis of different types of structured catalysts in function of the application. These types of catalysts are very promising for the conversion alcohols to olefins.

\section{Acknowledgements}

326 The authors want to express their thanks to the VITO personnel for their continuous support, especially R. Kemps, M. Mertens, I. Thijs, W. Bouwen (Sustainable Materials

328 Management Department, VITO). J. Lefevere thankfully acknowledges a PhD scholarship provided by VITO and the University of Antwerp. This work was done in the frame of a project of the fund for scientific research (FWO) in Belgium (G. 007113N).

\section{$\underline{\text { References }}$}

[1] B. Yilmaz, U. Müller, Topics in Catalysis (2009) 888.

[2] S. Ivanova, C. Lebrun, E. Vanhaecke, C. Pham-Huu, B. Louis, Journal of Catalysis 265 (2009) 1.

340 [5] J.Q. Chen, A. Bozzano, B. Glover, T. Fuglerud, S. Kvisle, Catalysis Today 106 (2005)

[3] M. Salmasi, S. Fatemi, A. Taheri Najafabadi, Journal of Industrial and Engineering Chemistry 17 (2011) 755.

342 [6] T. Ren, M. Patel, K. Blok, Energy 33 (2008) 817.

343 [7] D. Chen, K. Moljord, a. Holmen, Microporous and Mesoporous Materials 164 $344 \quad$ (2012) 239.

[8] D. Mores, E. Stavitski, M.H.F. Kox, J. Kornatowski, U. Olsbye, B.M. Weckhuysen, Chemistry (Weinheim an Der Bergstrasse, Germany) 14 (2008) 11320.

347 [9] J. Zhu, Y. Fan, N. Xu, Journal of Membrane Science 367 (2011) 14. 
348 [10] Z. Shan, W. Van Kooten, Microporous and Mesoporous Materials 34 (2000) 81.

349 [11] G. Seijger, O. Oudshoorn, Microporous and Mesoporous Materials 39 (2000) 195.

350 [12] B. Louis, F. Ocampo, H.S. Yun, J.P. Tessonnier, M.M. Pereira, Chemical Engineering $351 \quad$ Journal $161(2010) 397$.

352 [13] H. Yang, Z. Liu, H. Gao, Z. Xie, Journal of Materials Chemistry 20 (2010) 3227.

353 [14] S. Ivanova, E. Vanhaecke, L. Dreibine, B. Louis, C. Pham, C. Pham-huu, Applied

[15] J. Yao, C. Zeng, L. Zhang, N. Xu, Materials Chemistry and Physics 112 (2008) 637.

[16] I. Perdana, D. Creaser, J. Lindmark, J. Hedlund, Journal of Membrane Science 349 (2010) 83.

[17] A. Zampieri, S. Kullmann, T. Selvam, J. Bauer, W. Schwieger, H. Sieber, T. Fey, P. Greil, Microporous and Mesoporous Materials 90 (2006) 162.

360 [18] J.M. Zamaro, E.E. Miró, Chemical Engineering Journal 165 (2010) 701.

361 [19] J. Zamaro, M. Ulla, E. Miro, Chemical Engineering Journal 106 (2005) 25.

362 [20] S. Ivanova, B. Louis, B. Madani, J.P. Tessonnier, M.J. Ledoux, J. Phys. Chem. (2007) 4368.

364 [21] Y. Jiao, C. Jiang, Z. Yang, J. Zhang, Microporous and Mesoporous Materials 162

[22] J.N. Stuecker, J.E. Miller, R.E. Ferrizz, J.E. Mudd, J. Cesarano, Industrial \& Engineering Chemistry Research 43 (2004) 51.

368 [23] J. Stuecker, R. Ferrizz, J.C. III, J. Miller, Ind.\& Eng. Chem. Res. 44 (2005) 302.

369 [24] J. a. Lewis, J.E. Smay, J. Stuecker, J. Cesarano, Journal of the American Ceramic $370 \quad$ Society 89 (2006) 3599.

371 [25] J. Van Noyen, A. Wilde, M. Schroeven, S. Mullens, J. Luyten, International Journal 372 of Applied Ceramic Technology 9 (2012) 902.

373 [26] K.F. Leong, C.M. Cheah, C.K. Chua, Biomaterials 24 (2003) 2363.

374 [27] J. Luyten, S. Mullens, I. Thijs, KONA Powd. Part. J 28 (2010) 131. 
[28] M. Rombouts, S. Mullens, J. Luyten, in:, et al. P. J., da Silva Bartolo (Ed.), Innovative Developments in Design and Manufacturing, CRC Press, 2010, pp. 453457.

[29] M. Ravelingien, A.-S. Hervent, S. Mullens, J. Luyten, C. Vervaet, J.P. Remon, Applied Surface Science 256 (2010) 3693.

[30] J. Valli, Journal of Vacuum Science \& Technology A: Vacuum, Surfaces, and Films 4 (1986) 3007.

[31] Z. You, G. Liu, L. Wang, X. Zhang, Microporous and Mesoporous Materials 170 (2013) 235.

[32] V. Sebastián, O. de la Iglesia, R. Mallada, L. Casado, G. Kolb, V. Hessel, J. Santamaría, Microporous and Mesoporous Materials 115 (2008) 147.

[33] C. Brinker, G. Frye, A. Hurd, C. Ashley, Thin Solid Films 201 (1991) 97.

[34] C. Agrafiotis, A. Tsetsekou, Journal of the European Ceramic Society 20 (2000) 815.

[35] S. Zhang, Z. Gao, S. Liu, 34 (2012) 338.

[36] F. Patcas, Journal of Catalysis 231 (2005) 194.

\section{Figure captions}

Figure $1 \mathrm{Effect}$ of $\mathrm{SiO}_{2}$ concentration on the adhesion of the coating.

Figure 2 Effect of silica addition on the rheology of the coating suspension.

Figure $3 \mathrm{Effect}$ of milling and combination of milling and silica addition on the adhesion.

Figure 4 (a) 3DFD structure, (b) 1-1 (top) and 1-3 stacking (bottom) of 3DFD structure, (c) Cross section of fiber coated with the optimized coating method.

Figure 5 Hydrocarbon reagents pool (methanol + DME) conversion as function of WHSV for different coated structures and packed bed at $350^{\circ} \mathrm{C}$.

Figure 6 Yield of ethylene and propylene as function of WHSV for different coated structures and packed bed at $350^{\circ} \mathrm{C}$.

Figure 7 Ethylene + propylene selectivity as function of conversion for different coated structures and packed bed at $350^{\circ} \mathrm{C}$. 


\section{Table caption}

405 Table 1 Samples used in the catalytic testing.

406 Table 2 Results of catalytic conversion of methanol at $250^{\circ} \mathrm{C}$ and a WHSV of $4.56 \mathrm{~h}^{-1}$ for

407 different catalytic structures and packed bed of catalyst. 